\title{
INFLUÊNCIA DO AMBIENTE NA ESTRUTURA DE TAMANHO E BIOMASSA DA EMERITA BRASILIENSIS (SCHMITT, 1935) NA PRAIA BRAVA E GUARDA DO EMBAÚ
}

\author{
SILVA NETO, J. X. ${ }^{1 *}$; SIMPLICIO, A. ${ }^{1}$; DEUCHER, K. ${ }^{1}$; CUNHA, N. J. R. ${ }^{1}$ \& ALMEIDA, T. C. M. ${ }^{1}$
}

\author{
1. Universidade do Vale do Itajaí - UNIVALI.
}

*Autor correspondente: z.xavier@hotmail.com

\begin{abstract}
Silva Neto,J.X.,Simplicio,A., Deucher,K.,Cunha,N.J.R. \&Almeida,T.C.M.(2019). Influence oftheenvironmentonthesizeand biomass of Emerita brasiliensis (Schmitt, 1935) in Praia Brava and Guarda do Embaú. Braz. J. Aquat. Sci. Technol. 23(1). eISSN 1983-9057. DOI: 15233/bjast.v23n1. Emerita brasiliensis is an important species of Brazilian macrofauna, they are inhabitants of the intertidal areas, and have important components that enable their use as biological indicators environmental. Therefore, the objective was to analyze the relationship between population and size of individuals of Eremita brasiliensis, comparing two beaches on the coast of Santa Catarina, being Praia brava in Itajaí, with an increasing level of urbanization and Guarda do Embaú Beach in Paulo Lopes, which has a stationary degree. The collections took place in March and September 2016, with the sample area parallel to the sand and intertidal strips for a systematic sampling observing the estimated time for low tide. For collection, a $20 \mathrm{~cm}$ diameter PVC cylinder was used and buried at a depth of $20 \mathrm{~cm}$. The samples were arranged in a $0.5 \mathrm{~mm}$ opening mesh and sent to the laboratory, where the organisms of the genus Emerita brasiliensis were separated and identified for the measurement of biomorphometric data, observing the lateral diameter and rostro-caudal length of the carapace. The biomass weighing was performed before and after the drying procedure, performed in a controlled temperature oven at $37^{\circ} \mathrm{C}$ for 48 hours. For statistical analysis, the ANOVA test was used. Based on the individuals collected, it is observed Guarda do Embaú beach with more individuals when compared to Brava Beach. Statistical data show a significant difference between the months and the beaches, which is justified by the state of conservation of Guarda do Embaú beach, which remains static due to its low urbanization. Therefore, it is concluded that the difference between Emerita brasiliensis communities is due to changes in local dynamics, such as anthropic trampling and sedimentchange. The results were notaccurate due to the low number of samples, butare significantand relevantforfurther research.
\end{abstract}

Key Words: Benthic macrofauna, Sandy beach, Sediment, Urban influence, Bioindicators.

\section{INTRODUÇÃO}

As praias arenosas oceânicas constituem um dos ambientes mais dinâmicos da superfície do planeta (Alves et al., 2010). São ecossistemas costeiros onde as ondas, correntes e marés retrabalham ativamente o sedimento, o qual é composto por uma variedade de tipos e tamanhos de partículas, como areias grossas e areais finas (Viana et al., 2005; Mazzer \& Dillenburg, 2009; Alves et al., 2010).

A região entremarés de uma praia arenosa tem como principal característica biológica à presença de uma diversa e adaptada biota (Viana et al., 2005), sendo a distribuição e diversidade destes organismos determinada por fatores físicos, destacando-se a ação das ondas, tamanho das partículas de sedimento e declividade da praia (McLachlan, 1983).

Sendo o ambiente costeiro dominante na maioria das regiões tropicais e temperadas (Defeo \& McLachlan, 2005), as praias arenosas são um dos ambientes marinhos mais acessíveis ao homem e locais muito populares, consequentemente, é o ambiente costeiro mais utilizado pela população humana (Klein et al., 2004) causando mudanças antropogênicas que podem ter efeitos significativos na estrutura e no funcionamento das comunidades de uma praia (Amaral et al., 2016).
Embora diversos autores tenham destacado a importância das interações biológicas no controle da estrutura (Defeo \& Alava, 1995; Defeo, 1996; Defeo \& Martínez, 2003) e distribuição (Alava \& Defeo, 1991; Alves \& Rodrigues, 2003; Alves et al., 2010), a macrofauna bentônica das praias arenosas sofrem diretamente com os impactos e a dinâmica destas, pois possuem baixa motilidade e não são capazes de deixar rapidamente o local habitado (Alves et al., 2010).

A macrofauna de praias arenosas inclui a grande maioria dos táxons de invertebrados, com destaque especial para moluscos, crustáceos e poliquetas (Viana et al., 2005). A espécie Emerita brasiliensis, também conhecida como "Tatuí" ou "Tatuíra", é uma espécie importante da macrofauna brasileira, são habitantes das zonas entremarés, e possuem componentes relevantes que possibilitam o seu uso como bioindicadores ambientais, como por exemplo, sua ampla distribuição geográfica, respostas fisiológicas à poluentes químicos, fácil captura e sensibilidade a atividades humanas (Lercari \& Defeo, 1999; Fuentes et al.,2011).

Desta forma, foi realizado um estudo com o objetivo de analisar a influência do ambiente na estrutura de tamanho e biomassa dos indivíduos de Eremita brasiliensis em diferentes praias na costa 
Catarinense, considerando a hipótese de que em locais menos perturbados antropicamente, os indivíduos terão maior tamanho e biomassa.

\section{MATERIAIS E MÉTODOS}

\section{Procedimento de Campo}

Aárea de estudo correspondeu à porção arenosa das praias Brava $\left(-26.95^{\circ} \mathrm{S},-48.63^{\circ} \mathrm{O}\right)$ e Guarda do Embaú $\left(-27.90^{\circ} \mathrm{S},-48.59^{\circ} \mathrm{O}\right)$, localizadas nos municípios de Itajaí e Palhoça (SC) respectivamente, sendo que a Praia Brava possui um nível crescente de urbanização enquanto a praia da Guarda do Embaú possui um grau de urbanização estacionário.

As coletas foram efetuadas nos meses de março e setembro de 2016 em uma área de 30 metros de comprimento na zona entre marés com horário previsto para a maré baixa na área da amostragem.

Em uma extensão de 30 metros de comprimento, foram demarcados dez pontos amostrais na faixa de areia com três metros de distância entre si, sendo três aleatoriamente escolhidos para a realização de uma amostragem sistemática, onde foram obtidos dez pontos equidistantes ao longo do mesmo partindo do limite supralitoral em direção ao mar (Figura 1.A). Para tanto, foi necessário o uso de um amostrado cilíndrico de PVC de $20 \mathrm{~cm}$ de diâmetro $\mathrm{com} 20 \mathrm{~cm}$ de altura (Figura 1.B).

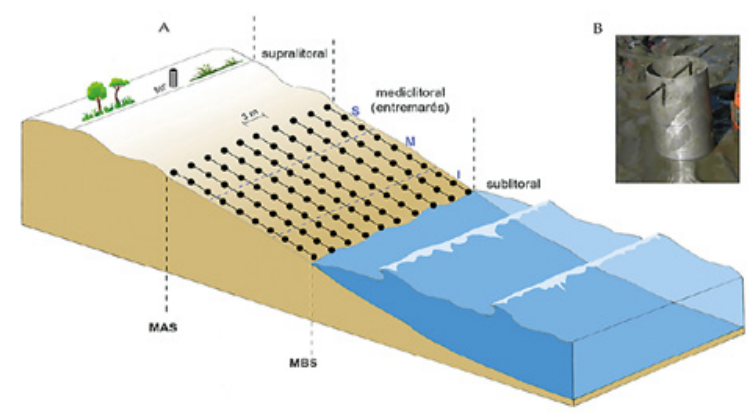

Figura 1 - A - Desenho amostral para coleta da macrofauna bentônica entremarés nos níveis superior $(\mathrm{S})$, médio $(\mathrm{M})$ e inferior $(\mathrm{I})$; B-Amostrador (20cm de diâmetro). Fonte: Rosa Filho, J. S. etal., 2015.

O sedimento amostrado foi lavado e peneirado em tela de nylon de $0,5 \mathrm{~mm}$ de abertura e o material retido foi acondicionado em sacos plásticos sendo devidamente etiquetados.

\section{Procedimento Laboratorial, Coleta e Análise dos Dados}

Após a coleta, as amostras foram encaminhadas até o laboratório de Ecologia de Comunidades Aquáticas, EMCT-UNIVALI, onde foram separados e identificados os organismos do gênero E. brasiliensis sendo acondicionados para a mensuração de dados biomorfométricos.

Utilizando-se um paquímetro, foi registrado o diâmetro lateral (DL) e comprimento rostro-caudal da carapaça (CRC) em milímetros. A pesagem da biomassa foi realizada, sendo registrada em gramas, antes e após o procedimento de secagem, onde contou com o auxílio de uma estufa com temperatura controlada à $37^{\circ} \mathrm{C}$ por 48 horas.

Para obtenção dos dados bioestatísticos foi utilizado o RStudio, fazendo o uso de histograma simples e com log das variáveis para obter a distribuição de frequência. Para testes de normalidade foi utilizado o método de Shapiro-Wilk e para teste de igualdade das variâncias (ANOVA multivariada) o método de Bartlett.

\section{RESULTADOS}

Com base nas amostras, foram identificados 135 exemplares de E. brasiliensis, sendo na Praia Brava coletados 54 espécimes (16em marçoe 38 em setembro), enquanto na praia da Guarda do Embaú foram obtidos 81 indivíduos (53 em março e 28 em setembro).

$\mathrm{Na}$ tabela 1 estão apresentadas as médias dos dados biomorfométricos e da biomassa dos exemplares coletados em ambas as praias nos respectivos meses, onde se tem o diâmetro lateral (DL), comprimento rostro-caudal da carapaça (CRC) em milímetros, peso úmido obtido através da pesagem realizada anteriormente ao processo de secagem e peso seco após os indivíduos serem secos em estufa sendo representados em gramas.

Tabela 1 - Média dos dados biomorfométricos e de biomassa dos exemplares de Emerita brasiliensis coletados na zona entremarés das praias Brava e Guarda do Embaú nos meses de março e setembrode 2016 e do total dos indivíduos coletados.

\begin{tabular}{|c|c|c|c|c|c|c|c|c|}
\hline \multicolumn{5}{|c|}{ Praia Brava } & \multicolumn{4}{|c|}{ Guarda do Embaú } \\
\hline & $\begin{array}{c}\mathrm{DL} \\
(\mathrm{mm})\end{array}$ & $\begin{array}{l}\text { CRC } \\
(\mathrm{mm})\end{array}$ & $\begin{array}{c}\text { Peso } \\
\text { Úmido(g) }\end{array}$ & $\begin{array}{c}\text { Peso } \\
\text { Seco(g) }\end{array}$ & $\begin{array}{c}\mathrm{DL} \\
(\mathrm{mm})\end{array}$ & $\begin{array}{l}\text { CRC } \\
(\mathrm{mm})\end{array}$ & $\begin{array}{c}\text { Peso } \\
\text { Úmido(g) }\end{array}$ & $\begin{array}{c}\text { Peso } \\
\text { Seco(g) }\end{array}$ \\
\hline Mar. & 12 & 24 & 2,14 & 0,51 & 7,9 & 15,3 & 0,57 & 0,09 \\
\hline Set. & 3,9 & 8,29 & 0,31 & 0,06 & 5,9 & 12,3 & 0,47 & 0,09 \\
\hline Total & 6,3 & 12 & 0,85 & 0,19 & 7,2 & 14 & 0,5 & 0,09 \\
\hline
\end{tabular}

A partir destes dados, foram gerados os histogramas de barras, onde observa-se gráficos bimodais, sendo causado pordimorfismo sexual, variação nos meses, pois as coletas ocorreram em estações diferentes, ou um sistema de hierarquia naespécie. Sendo assim, os histogramas não mostraram normalidade na frequência do organismo em ambas as praias.

Após, realizou-se o teste de normalidade de Shapiro-Wilk (tabela 2), com o objetivo de fornecer uma estatística para avaliar se a amostra tem distribuição normal. No qual se rejeita h0, pois os valores obtidos foram menores que 0,05 ( $\alpha=0,05)$, dando a evidência de algo fora da normalidade.

Com isso, foi realizado a estatística de teste de Bartlett, onde obteve-se o p-Value maior do que o de seu nível de a $(0,05)$, aceitando a hipótese nula de que as variâncias são iguais (tabela 3 ).

Para obtenção da significância dos dados, realizou-se o teste ANOVA Multivariada, obtendo o 
p-Value: 0.01105 , podendo assim, concluir que há diferença significativa entre as praias.

Tabela 2 - Resultado referente ao teste de Shapiro e seus respectivos p-Value.

\begin{tabular}{cc} 
DL março & $1,18 \mathrm{E}-02$ \\
DL setembro & $7,03 \mathrm{E}-08$ \\
CRC março & $2,44 \mathrm{E}-03$ \\
CRC setembro & $1,50 \mathrm{E}-07$ \\
Peso.úmido março & $9,46 \mathrm{E}-08$ \\
Peso.úmido setembro & $4,99 \mathrm{E}-12$ \\
Peso.seco março & $7,79 \mathrm{E}-09$ \\
Peso.seco setembro & $3,18 \mathrm{E}-12$ \\
\hline
\end{tabular}

Tabela 3 - Referente ao teste de Bartlett onde a hipótese é aceita.

\begin{tabular}{cc}
\hline $\mathrm{DL}$ & 0.5002 \\
$\mathrm{CRC}$ & 0.7833 \\
Peso úmido & 0.5231 \\
Peso seco & 0.1599 \\
\hline
\end{tabular}

\section{DISCUSSÃO}

Considerando a estatística apresentada, onde a biomassa e tamanho populacional da Praia Brava foi menor, pode-se concluir que ocorre diferença na comunidade de Emerita brasiliensis entre as duas praias arenosas, sendo possivelmente ocasionada pela alteração na dinâmica local, que é provocada pelo crescimento da urbanização que atinge a Praia Brava, sendo que na praia da Guarda do Embaú a ação antrópica é menor, pois o estado de conservação se mantém estático.

Santos Jr. ePereira (2011)demonstram quea Praia Brava sofre um acelerado processo de transformação socioespacial e o crescimento da urbanização é visível, registrando-se já um início de verticalização das construções em resposta às pressões por falta de espaço e pela valorização imobiliária.

Em contrapartida, segundo Cardoso (2018), a Praia da Guarda do Embaú está situada em uma restinga e na confluência de três unidades de conservação, áreas tomadas pela UNESCO como Reserva da Biosfera da Mata Atlântica, sendo elas, o Parque Estadual da Serra do Tabuleiro, a Área de Proteção Ambiental (APA) do Entorno Costeiro e a APA da Baleira Franca. Portando, com uma menor influência antrópica.

Dessa forma, a hipótese de que em ambientes menos perturbados antropicamente resultaria em maiores tamanhos médios de tatuíras foi aceita, além de resultar em maior tamanho populacional, enquanto a hipótese de se obter organismos com biomassa elevada foi rejeitada.

Fuentes e colaboradores (2011) demonstraram que organismos de praias dissipativas apresentam biomassa mais elevada, além de que possivelmente apenas indivíduos mais desenvolvidos consigam permanecer sob condições excessivas de perturbação física ou resultante de ação humana, como por exemplo pisoteio antrópico e mudança no sedimento, sendo que a Praia Brava, devido ao aumento da construção civil, possui um sedimento mais fino e argiloso, visto que para o sucesso destes organismos, o ambiente necessita de um sedimento mais grosso, como em praias reflexivas.

\section{CONCLUSÃO}

Os resultados não foram precisos devido ao baixo número de amostras, uma vez que a coleta ocorreu apenas em 2016. No entanto, são significativos e relevantes para novas pesquisas, as quais necessitam de uma escala temporal maior e o registro de alguns parâmetros, como os períodos de maior e menor uso pelos humanos e outras variáveis ambientais do local, além de mais artigos contendo tais objetivos, para que assim, a análise tenha mais credibilidade.

\section{REFERÊNCIAS}

Alava, A. \& Defeo, O. 1991. Distributional pattern and population dynamics of Excirolana armata (Isopoda: Cirolanidae) in a Uruguayan sandy beach. Estuar. Coast. Shelf Sci. 33(5): 433-444.

Alves, E.S., Rodrigues, S.A., Pezzuto, P.R., \& Shimizu, G.Y. 2010. Caracterização ambiental da porção subaérea de praias arenosas expostas com diferentes características morfodinâmicas no litoral centro-norte de Santa Catarina, Brasil. Braz. J. Aq. Sci. and Tech. 8(1): 1-24.

Alves, E.S \& Rodrigues, S.A. 2003. Distributional Patterns of Austinixa Patagoniensis (Rathbun, 1918) (Decapoda: Pinnotheridae), A Symbiotic Crab of" Callichirus Major" (Decapoda: Calianassidae), in Balneário Camboriú Beach, Santa Catarina, Brazil. J. Coast. Res. 35: 408-417.

Amaral, A.; Corte, G.N.; Filho, J.S.R.; Denadai, M.R.; Colling, L.A.; Borzone, C.; Veloso, V.; Omena, E.P.; Zalmon, I.R.; Rocha-Barreira, C.A.; Souza, J.R.B.; Rosa, L.C. \& Almeida, T.C.M. 2016. Brazilian sandy beaches: characteristics, ecosystem services, impacts, knowledge and priorities. Braz. J. Oceanog. 64(SPE2): 5-16.

Cardoso, E.W. 2018. Caracterização do turismo no território da APA da Baleia Franca. In: ICMBio. (ed) Plano de manejo - Área de proteção ambiental da Baleia Franca. Anexo 19.

Defeo, O. 1996. Experimental management of an exploited sandy beach bivalve population. Rev. Chil. Hist. Nat. 69: 605-614. 
Defeo, O. \& Alava, A. 1995 Effects of human activities on long-term trends in sandy beach populations: the wedge clam Donax hanleyanus in Uruguay. Mar. Ecol. Prog. Ser. 123: 73-82

Defeo, O. \& Martínez, G. 2003. The habitat harshness hypothesis revisited: life history of the isopod Excirolana braziliensis in sandy beaches with contrasting morphodynamics. J. of the Mar. Bio. Ass. of the U.K. 83(2): 331-340.

Defeo, O. \& McLachlan, A. 2005. Patterns, processes and regulatory mechanisms in sandy beach macrofauna: a multi-scale analysis. Mar. Ecol. Prog. Ser. 295: 1-20.

Fuentes, E.V., Schulze, B., Saito, E.N., Tsuda, É.T., Saldanha, J.H., Silva, I.K. \& Daura-Jorge, F.G. 2011. A Influência do Ambiente no Tamanho e Bomessa da Tatuíra (Emerita brasiliensis) na Ilha de Santa Catarina, Brasil. Est. Eco. na Ilha de SC. 221-228.

Klein, Y.L.; Osleeb, J.P. \& Viola, M.R. 2004. Tourismgenerated earnings in the coastal zone: a regional analysis. J. Coast. Res. 20(4 (204)): 1080-1088.

Lercari, D. \& Defeo, O. 1999. Effects of freshwater discharge in sandy beach populations: the mole crab Emerita brasiliensis in Uruguay. Estuar. Coast. Shelf Sci. 49(4): 457-468.

Mazzer, A. \& Dillenburg, S. 2009. Variações temporais da linha de costa em praias arenosas dominadas por ondas do sudeste da Ilha de Santa Catarina (Florianópolis, SC, Brasil). Pesq. em Geociências. 36(1): 117-135.

McLachlan, A. 1983. Sandy beach ecology - a review. In: McLachan A. \& Erasmus T. (eds) Sandy beaches as ecosystems. Dordrecht: Springer. 19: 321-380.

Rosa Filho, J.S.; Corte, G.N.; Maria, Colling, L.A.; Denadai, M.R.; Rosa, L.C.; Borzone, C.A.; Almeida, T.C.M.; Zalmon, I.R.; Omena, E.; Veloso, V. \& Amaral, A.C.Z. 2015. Monitoramento de longo prazo da macrofauna bentônica entremarés de praias arenosas. In: Turra, A. \& Denadai, M.R. (ed.) Protocolos para o monitoramento de habitats bentônicos costeiros - Rede de Monitoramento de Habitats Bentônicos Costeiros - ReBentos. IOUSP. 194-208.

Santos Júnior, A. \& Pereira, R.M.F.A. 2011. As recentes transformações socioespaciais do litoral de Santa Catarina: o caso da Praia Brava - Itajaí, SC. Geosul. 26(5): 109-128.

Viana, M.G.; Rocha-Barreira, C.A. \& Grossi Hijo, C.A. 2005. Macrofauna bentônica da faixa entremarés e zona de arrebentação da praia de Paracuru (Ceará-Brasil). Braz. J. Aq. Sci. Tech. 9(1): 75-82.

Submetido: Setembro/19 Revisado: Julho/2020 Aceito:Julho/2020 Publicado: 05/10/2020 